\title{
Morbilidad neonatal de los prematuros tardíos en embarazos únicos: estudio de caso-control
}

\author{
Enrique Valdés $R .^{1}$, Alvaro Sepúlveda M. $^{1}$, Jorge Catalán M. ${ }^{2}$, Alvaro Reyes $P .{ }^{a}$ \\ 1 Unidad de Medicina Fetal, ${ }^{2}$ Unidad de Neonatología, Hospital Clínico de la Universidad de Chile, Santiago, Chile.
}

a Departamento de Bioestadística, Pontificia Universidad Católica de Valparaíso, Chile.

\section{RESUMEN}

Objetivo: Comparar los riesgos de morbilidad neonatal entre los prematuros tardíos (PT) y neonatos de término. Método: Estudio de caso control. Se revisan fichas clínicas de partos durante el año 2007. Se excluyen neonatos con malformaciones congénitas mayores, alteración neuromuscular, embarazos múltiples y aneuploidías. Los casos corresponden a todo PT nacido durante el período estudiado y los controles a nacidos de término en el mismo período. Los resultados neonatales fueron obtenidos y los riesgos calculados usando pruebas de Chi cuadrado y exacto de Fisher. Resultados: Se identifican 1536 partos, con una tasa de PT de 7,1\% (109 casos), 62 cumplieron con criterios de inclusión. El grupo control consistió en 124 partos de término. PT presentaron 2 veces más riesgo de cesárea $(p=0,0094)$ que los de término. El riesgo de ser admitido en UCIN fue de $88(p=0,000)$. Los riesgos de morbilidad neonatal fueron: SDR (OR 23; $p=0,000$ ), hipoglicemia (OR 6; $p=0,014$ ), hipocalcemia (OR 6; $p=0,014$ ), hiperbilirrubinemia (OR 28; $p=0,000)$ y necesidad de fototerapia (OR 23; $p=0,000)$. No hubo diferencias en la presentación de enterocolitis necrotizante $(p=0,478)$ ni sepsis neonatal $(p=0,615)$. La mortalidad neonatal fue significativamente superior en los PT $(p=0,044)$. Conclusión: Los PT deben ser considerados de alto riesgo en el período neonatal. Nuestros resultados son importantes para tomar decisiones clínicas respecto al mejor momento de finalizar un embarazo con riesgo inminente de prematurez.

\section{PALABRAS CLAVE: Prematuro tardío, síndrome de distrés respiratorio, mortalidad, morbilidad neonatal, resultado perinatal}

\section{SUMMARY}

Objective: To compare neonatal morbidity risks between late preterm (LP) and term deliveries. Methods: Case control study. Medical records in 2007 were reviewed. Major congenital malformations, neuromuscular handicap, twin pregnancies and aneuploidies were excluded. The Study group corresponds to all LP births during that period and the control group to term deliveries in the same period. Neonatal outcomes were collected and different risks were calculated using Chi square test and Fisher exact tests. Results: 1536 deliveries with a LP rate of $7.1 \%$ (109 cases) were observed, 62 cases met inclusion criteria. The control group consisted in 124 single term deliveries. LP had 2 times more risk of cesarean section $(p=0.0094)$ than term deliveries. The risk of NICU admission was $88(p=0.000)$. Neonatal morbidity risks were: RDS (OR 23, $p=0.000$ ), hypoglycemia (OR $6, p=0.014$ ), hypocalcaemia (OR $6, p=0.014$ ), hyperbillirrubinemia (OR $28, p=0.000)$ and phototherapy (OR 23, $p=0.000)$. There were no differences in necrotizing enterocolitis $(p=0.478)$ and risk of neonatal sepsis $(p=0.615)$. Neonatal mortality was significantly higher in LP babies 
$(p=0.044)$. Conclusion: LP newborn must be considered as high risk in the neonatal period. These results are important in making clinical decisions about the better time to end pregnancy.

\section{KEY WORDS: Late preterm, respiratory distress syndrome, mortality, neonatal morbidity, perinatal outcome}

\section{INTRODUCCIÓN}

En la actualidad se considera parto prematuro a todo embarazo que finaliza antes de las 37 semanas, basándose en una fecha de última regla (FUR) correcta (1) o una ecografía de primer trimestre. En países desarrollados los prematuros se han incrementado en alrededor de un 30\% los últimos 25 años, llegando incluso en algunos países a tasas de un $12 \%$ del total de nacimientos (2). Recientemente, la NICHD definió a los prematuros tardíos (PT) como todo nacimiento producido entre las semanas 34+0 y 36+6 desde la FUR (3) para de esta manera enfatizar el riesgo perinatal incrementado de este grupo de niños, los cuales previamente eran considerados y manejados como neonatos de bajo riesgo.

Los PT comprenden el $71-79 \%$ del total de prematuros (4-8) llegando en algunas series a tasas de $9,1 \%$ del total de los nacimientos $(9,10)$. En el Hospital Clínico de la Universidad de Chile los PT corresponden al $60 \%$ del total de prematuros, observando una ligera disminución los últimos cinco años debido al incremento de prematuros de edades gestacionales menores (11). Diversas publicaciones recientes han demostrado que los PT tienen resultados neonatales significativamente peores en comparación con recién nacidos de término $(3,4,6,10,12-16)$ siendo la morbilidad respiratoria la entidad más evaluada. Esta diferencia observada es también significativa en evaluaciones a largo plazo.

El objetivo de este estudio es comparar el riesgo de morbilidad neonatal entre un grupo de PT y recién nacidos de término en una Unidad de Neonatología de un hospital universitario.

\section{MATERIAL Y MÉTODO}

Este estudio de caso control realizado en la Unidad de Medicina Fetal y la Unidad de Neonatología del Departamento de Obstetricia y Ginecología Hospital Clínico Universidad de Chile. La base de datos electrónica neonatal fue revisada por uno de los autores (J.C.), identificando todos los partos en el año 2007. Posteriormente, se identifican todos los nacidos vivos, excluyendo aquellos con malformaciones congénitas mayores, discapacidad neuromuscular, embarazos múltiples y aneuploidías (con diagnóstico antenatal o postnatal). Se consideran como casos a todo niño nacido entre las $34+0$ y $36+6$ semanas desde la FUR. El grupo control fue conformado por niños de término nacidos antes y después de cada PT. Realizamos un seguimiento retrospectivo de ambos grupos hasta los 28 días de vida, obteniendo los resultados neonatales de la base de datos electrónica de la Unidad de Neonatología. Se registran todos aquellos neonatos que dentro de los primeros 28 días de nacimiento desarrollaron síndrome de distrés respiratorio (SDR), hipoglicemia, hipocalcemia, hiperbilirrubinemia, enterocolitis necrotizante, hipotermia, sepsis neonatal y aquellos que requirieron fototerapia, hospitalización en la Unidad de Cuidados Intensivos o Intermedio Neonatal (UCIN), o que murieron durante el primer mes de vida. Después de comparar ambos grupos, analizamos los resultados por semana. Las características demográficas maternas fueron obtenidas de las fichas clínicas o por información en la base de datos de la Unidad de Neonatología y de Medicina Fetal.

Definiciones.

Parto prematuro: todo embarazo que finaliza antes de la semana $37+0$ desde la FUR.

Prematuro tardío (PT): todo embarazo que finaliza entre las semanas $34+0$ y $36+6$ desde la FUR.

PT por indicación: todo embarazo interrumpido entre las semanas $34+0$ y $36+6$ debido a una causa materna o fetal.

PT espontáneo: todo embarazo que finaliza entre las semanas $34+0$ y $36+6$ desde la FUR, a causa de un inicio espontáneo del trabajo de parto o por rotura espontánea prematura de las membranas.

Las patologías neonatales fueron definidas como:

Hipoglicemia neonatal: recién nacido con glicemia bajo $50 \mathrm{mg} / \mathrm{dl}$.

Hipocalcemia neonatal: calcemia total menor a $7 \mathrm{mg} / \mathrm{dl}$

Enterocolitis necrotizante: recién nacido con intolerancia alimentaria, deposiciones con sangre y 
distensión abdominal después de la segunda semana de vida.

Mortalidad neonatal: muerte infantil de menos de 28 días de vida.

Hiperbilirrubinemia neonatal: incremento de bilirrubinemia por sobre $0,5 \mathrm{mg} / \mathrm{dl}$ por hora durante el primer día de vida, o valores de bilirrubina sérica que requiera fototerapia.

Sepsis neonatal: presencia de un síndrome de respuesta inflamatoria sistémica, con hemocultivos positivos en el período neonatal.

Síndrome de distrés respiratorio: presencia de taquipnea, taquicardia, quejido, cianosis, retracción subcostal y grados diversos de compromiso de la oxigenación en el recién nacido.

Hemorragia intraventricular: grado 1 (hemorragia de la matriz germinal); grado 2 (sangre intraventricular sin distensión del sistema ventricular); grado 3 (presencia de sangre llenando y distendiendo el sistema ventricular) y grado 4 (hemorragia con compromiso de parénquima ó infarto venoso periventricular).

Análisis estadístico.

Para comparar los resultados neonatales entre ambos grupos, se calcularon los distintos riesgos usando las pruebas de chi cuadrado y Fisher Exac- to para las variables categóricas. Para el análisis demográfico materno usamos la prueba de Kolmogorov-Smirnov para demostrar distribución normal de las variables continuas, usando la prueba de $t$ student, media y desviación estándar (DE) si presentaban distribución normal. Todos los análisis estadísticos fueron realizados con el programa SPSS v15.0, considerando como significativo un $p<0,05$.

\section{RESULTADOS}

En el período estudiado, identificamos 1536 partos únicos, correspondiendo 109 de ellos a PT (tasa 7,1\%). De este grupo, 62 cumplieron con los criterios de inclusión. El grupo control consistió en 124 recién nacidos de término de embarazos únicos. Las características demográficas maternas se muestran en la Tabla I. Las pacientes con un parto prematuro previo tuvieron un riesgo 11 veces mayor de presentar un PT en el embarazo actual que el grupo control $(p=0,008)$. Las patologías maternas están significativamente incrementadas en aquellas embarazadas que tuvieron un $\mathrm{PT}$, con un riesgo de casi 4 veces en comparación con las que tuvieron un parto de término, siendo la preeclampsia la patología principal (Tabla I).

Tabla I

CARACTERÍSTICAS DEMOGRÁFICAS DE MADRES DE NIÑOS NACIDOS A TÉRMINO Y PREMATURO TARDÍO

\begin{tabular}{|c|c|c|c|c|}
\hline Variable & $\begin{array}{l}\text { Casos } \\
(n=62)\end{array}$ & $\begin{array}{c}\text { Controles } \\
(n=124)\end{array}$ & $\begin{array}{c}\text { OR } \\
\text { (IC95\%) }\end{array}$ & Valor $p$ \\
\hline Edad materna media $( \pm \mathrm{DE})$ & $29,2( \pm 6,59)$ & $28,7( \pm 6,28)$ & - & $0,296^{*}$ \\
\hline IMC (kg/m2) media ( $\pm \mathrm{DE})$ & $17,5( \pm 10,9)$ & $20,7( \pm 10,5)$ & - & $0,275^{\star}$ \\
\hline Nuliparidad n (\%) & $31(50)$ & $63(50,8)$ & $0,9(0,5-1,8)$ & $0,917 \dagger$ \\
\hline Cesárea n (\%) & $41(66,13)$ & $57(45,97)$ & $2,3(1,2-4,6)$ & $0,0094 \dagger$ \\
\hline Enfermedad materna n (\%) & $30(48,39)$ & $25(20,16)$ & $3,8(1,9-7,9)$ & $0,000 \dagger$ \\
\hline CIE & $1(1,61)$ & $3(2,42)$ & $0,7(0,01-8,6)$ & $0,73+$ \\
\hline Diabetes gestacional & $7(11,29)$ & $12(9,68)$ & $1,2(0,4-3,6)$ & $0,68 \dagger$ \\
\hline Preeclampsia & $11(17,74)$ & $0(0)$ & $23,8 \ddagger$ & $0,0000 \dagger$ \\
\hline Trombofilia & $3(4,84)$ & $1(0,81)$ & $6,3(0,5-333,9)$ & $0,072 \dagger$ \\
\hline Desprendimiento placentario & $5(8,06)$ & $1(0,81)$ & $11(1,2-523,4)$ & $0,008+$ \\
\hline Parto prematuro previo n (\%) & $5(8,06)$ & $1(0,8)$ & $11(1,2-514,2)$ & $0,008 \dagger$ \\
\hline RPM n (\%) & $12(19,35)$ & $6(4,84)$ & $4,7(1,5-16,1)$ & $0,002 \dagger$ \\
\hline RCF n (\%) & $9(14,52)$ & $2(1,61)$ & $10,6(2,1-102,3)$ & $0,000 \dagger$ \\
\hline
\end{tabular}

IMC: índice de masa corporal; RPM: rotura prematura de membranas. CIE: colestasia intrahepática del embarazo. RCF: restricción del crecimiento fetal. DE: desviación estándar. ${ }^{*}=\mathrm{t}$ student; $\dagger=$ chi cuadrado; $\ddagger=$ intervalo de confianza no calculado por existir 0 casos de preeclampsia en grupo control. 
Luego de analizar el tipo de parto, los PT demostraron tener un riesgo dos veces mayor de cesárea que el grupo control, siendo esta diferencia significativa $(p=0,0094)$. El APGAR a los 5 minutos fue también significativamente menor, con un puntaje de 8,6 para los PT y de 9,2 para los nacidos de término $(p=0,000)$. En el periodo neonatal, los PT tuvieron 88 veces más riesgo de ser admitidos a UCIN que el grupo control $(p=0,000)$.

Las patologías neonatales para ambos grupos se presentan en la Tabla II. EI SDR, la hipoglicemia y la hiperbilirrubinemia fueron las entidades con los riesgos mayores en los PT en el período neonatal, con un OR de 23, 23 y 13 respectivamente. Sin embargo, no hubo diferencias en el riesgo de enterocolitis necrotizante ni sepsis neonatal. La mortalidad neonatal fue 4 veces mayor en los PT que en los nacidos de término (Tabla II).

Luego de analizar ambos grupos semana a semana, demostramos que la tasa de cesárea fue mayor en los PT hasta las 35 semanas, pero a edades gestacionales mayores las tasas fueron similares (Tabla III). El riesgo de SDR y de requerir ingreso a UCIN fue inversamente relacionado con la edad gestacional, con una reducción importante desde las 37 semanas (Figura 1).

La severidad de la hiperbilirrubinemia disminuyó significativamente con el incremento de la edad gestacional, con una necesidad de fototerapia de $75 \%$ y $19,8 \%$ a las 34 y 36 semanas respectivamente. Después de las 36 semanas, ningún neonato con hiperbilirrubinemia requirió fototerapia (Tabla III).

\section{DISCUSIÓN}

Los resultados obtenidos en nuestro estudio confirman que los PT deben ser considerados como niños de alto riesgo en el período neonatal. La morbilidad respiratoria en nuestros resultados es consistente con datos publicados en estudios internacionales $(3,10)$. Esto también se aplica a morbilidades metabólicas como la hipoglicemia y la hipocalcemia.

Nuestra incapacidad en demostrar una diferencia significativa entre ambos grupos en el riesgo de desarrollar enterocolitis necrotizante es debido a la baja incidencia de esta patología, siendo necesario incrementar el número de pacientes a estudiar.

Tabla II

COMPARACIÓN DE LA MORBILIDAD NEONATAL DE ACUERDO A LA EDAD GESTACIONAL AL PARTO

\begin{tabular}{|c|c|c|c|c|}
\hline Evento & $\begin{array}{l}\text { PPT } \\
\text { n (\%) }\end{array}$ & $\begin{array}{l}\text { PT } \\
\text { n (\%) }\end{array}$ & $\begin{array}{c}\text { OR } \\
(\mathrm{IC} 95 \%)\end{array}$ & Valor $p$ \\
\hline Admisión UCIN & $26(41,9)$ & $1(0,8)$ & $\begin{array}{c}88,8 \\
(13,3-3669,5)\end{array}$ & 0,000 \\
\hline SDR & $11(17,7)$ & $0(0)$ & $23,4^{*}$ & 0,000 \\
\hline Hiperbilirrubinemia & $19(30,6)$ & $4(3,2)$ & $\begin{array}{c}13,2 \\
(4,0-55,7)\end{array}$ & 0,000 \\
\hline Admisión fototerapia & $11(17,7)$ & $0(0)$ & $23,4^{*}$ & 0,000 \\
\hline Hipoglicemia & $3(4,8)$ & $0(0)$ & $6^{*}$ & 0,014 \\
\hline Hipocalcemia & $3(4,8)$ & $0(0)$ & $6,1^{*}$ & 0,014 \\
\hline Hipotermia & $0(0)$ & $1(0,8)$ & $0,5^{\star}$ & 0,478 \\
\hline Enterocolitis necrotizante & $0(0)$ & $1(0,8)$ & $0,5^{\star}$ & 0,478 \\
\hline Mortalidad neonatal & $2(3,2)$ & $0(0)$ & $4^{*}$ & 0,044 \\
\hline Sepsis neonatal & $1(1,6)$ & $1(0,8)$ & $\begin{array}{c}2 \\
(0,02-159,5)\end{array}$ & 0,615 \\
\hline Hemorragia intraventricular & $1(1,61)$ & $0(0)$ & $2,01^{*}$ & 0,156 \\
\hline
\end{tabular}

PPT: parto prematuro tardío. PT: parto de término. UCIN: Unidad de Cuidados Intensivos Neonatal. SDR: síndrome de distrés respiratorio. *: intervalo de confianza no calculado por presentar algunos de los grupos 0 casos. 
Tabla III

FRECUENCIA DE EVENTOS PERINATALES DE ACUERDO A LA EDAD GESTACIONAL AL PARTO POR SEMANA

\begin{tabular}{lrrrrrrc}
\hline Variable $n(\%)$ & $\begin{array}{r}34 \text { sem } \\
(n=15)\end{array}$ & $\begin{array}{r}35 \text { sem } \\
(n=18)\end{array}$ & $\begin{array}{r}36 \text { sem } \\
(n=29)\end{array}$ & $\begin{array}{r}37 \text { sem } \\
(n=15)\end{array}$ & $\begin{array}{r}38 \text { sem } \\
(n=39)\end{array}$ & $\begin{array}{r}39 \text { sem } \\
(n=44)\end{array}$ & $\begin{array}{r}40-41 \text { sem } \\
(n=26)\end{array}$ \\
\hline SDR & $4(26,7)$ & $4(22,2)$ & $3(10,3)$ & $0(0)$ & $0(0)$ & $0(0)$ & $0(0)$ \\
Hipoglicemia & $1(6,7)$ & $1(5,5)$ & $1(3,4)$ & $0(0)$ & $0(0)$ & $0(0)$ & $0(0)$ \\
Hiperbilirrubinemia & $8(53,3)$ & $6(33,3)$ & $5(17,2)$ & $1(6,7)$ & $3(7,7)$ & $0(0)$ & $0(0)$ \\
Fototerapia & $6(40)$ & $4(22,2)$ & $1(3,4)$ & $0(0)$ & $0(0)$ & $0(0)$ & $0(0)$ \\
Cesárea & $11(73,3)$ & $15(83,3)$ & $15(51,7)$ & $5(33,3)$ & $23(59)$ & $17(38,6)$ & $12(46,1)$ \\
\hline
\end{tabular}

SDR: síndrome de distrés respiratorio.

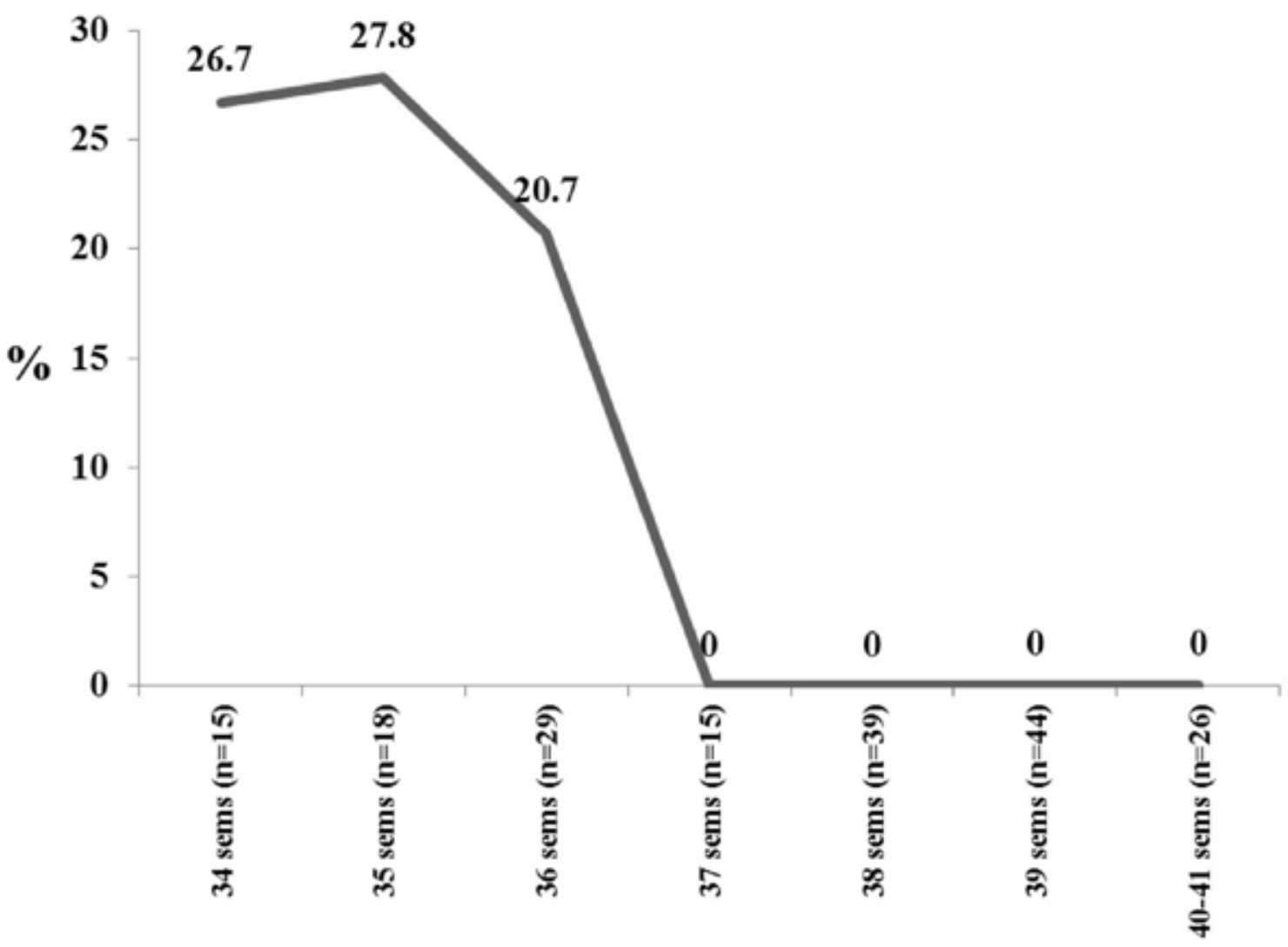

Edad Gestacional (semanas)

Figura 1. Tasas de morbilidad respiratoria compuesta según la edad gestacional al parto. 
El riesgo incrementado de cesárea es probablemente atribuido a los PT por indicación. Destaca la alta tasa de cesárea en el grupo control, sin embargo, las cifras son similares a la tasa nacional chilena de $42 \%$ (17) que desafortunadamente es una de las más altas del mundo. En el análisis no hicimos diferencia entre los PT espontáneos y por indicación, pero diversas publicaciones recientes han demostrado que los PT por indicación tienen un mayor resultado neonatal adverso y tasas de cesárea más altas que los espontáneos (18). Sin embargo, los datos publicados son contradictorios en este punto (19).

Nuestros resultados demostraron que la preeclampsia es la principal patología materna en las embarazadas que presentaron un PT, siendo probablemente esta razón la causa del resultado neonatal adverso identificado. En un futuro nuestra Unidad desarrollará un estudio para comparar exclusivamente a los PT de acuerdo a la causa (espontáneo versus por indicación) para objetivar la diferencia en la incidencia de morbilidad respiratoria, y así concentrar las estrategias de prevención en el grupo de mayor riesgo.

La debilidad de nuestro estudio es su naturaleza retrospectiva y el tamaño muestral reducido, por lo que estudios locales prospectivos son necesarios para validar nuestros resultados.

Consideramos que nuestro estudio es importante para tomar decisiones clínicas con respecto al mejor momento para finalizar el embarazo cuando es posible diferir la interrupción, y de esta manera en un futuro identificar herramientas útiles en diferir el parto o incrementar los resultados neonatales. El manejo de embarazos en riesgo de finalizar en PT debe ser un trabajo en equipo que involucre a obstetras, neonatólogos, perinatólogos y matronas.

\section{CONCLUSIÓN}

Es fundamental reforzar en las unidades de maternidad y neonatología el concepto de que los PT son un grupo de alto riesgo no solo en el período neonatal, sino que también a largo plazo.

\section{REFERENCIAS}

1. Engle W. A recommendation for the definition of "late preterm" (Near-Term) and the birth weight-gestational age classification system. Semin Perinatol 2006;30:27.

2. Davidoff M, Dias Todd, Damus K, Russell R, Bettegowda V, Dolan S, et al. Changes in the gestational age distribution among US singleton births: impact on rates of late preterm birth, 1992 to 2002. Semin Perinatol 2006;30:8-15.

3. Kalyoncu O, Aygun C, Cetinoglu E, Kucukoduk S. Neonatal morbidity and mortality of late-preterm births. J Matern Fetal Neonatal Med 2010;23:607-12.

4. Bastek JA, Sammel MD, Rebele EC, Srinivas S, Elovitz $M$. The effects of a preterm labor episode prior to 34 weeks are evident in late preterm outcomes, despite the administration of betamethasone. Am J Obstet Gynecol 2010;203:140.e1-7.

5. Goldenberg R, Culhane J, lams J, Romero R. Preterm birth 1. Epidemiology and causes of preterm birth. Lancet 2008;371:75-84.

6. Dani C, Corsini I, Piergentili L, Bertini G, Pratesi S, Rubaltelli FF. Neonatal morbidity in late preterm and term infants in the nursery of a tertiary hospital. Acta Paediatrica 2009;98:1841-3.

7. McIntire D, Leveno KJ. Neonatal mortality and morbidity rates in late preterm births compared with births at term. Obstet Gynecol 2008;111:35-41.

8. Demestre X, Raspall F, Martínez-Nadal S, Vila C, Elizari MJ, Sala P. Prematuros tardíos: una población de riesgo infravalorada. An Pediatr (Barc) 2009;71:291-8.

9. Reddy U, Wen Ko C, Raju T, Willinger M. Delivery indications at late-preterm gestations and infant mortality rates in the United States. Pediatrics 2009;124:234-40.

10. The Consortium on Safe Labor. Respiratory morbidity in late preterm births. JAMA 2010;304:419-25.

11. Sepúlveda A, Köbrich S, Guiñez R, Hasbún J. Morbilidad de prematuros tardíos: evidencia actual y nuevo enfoque. Rev Chil Obstet Ginecol 2012;77:154-8.

12. Meloni A, Antonelli A, Deiana S, Rocca A, Atzei A, Paoletti $A$, et al. Late preterm: obstetric management. J Matern Fetal Neonatal Med 2010;23(Suppl 3):113-5.

13. Melamed N, Klinger G, Tenenbaum K, Herscovici T, Linder N, Hod M, et al. Short-term neonatal outcome in low-risk, spontaneous, singleton, late preterm deliveries. Obstet Gynecol 2009;114:253-60.

14. Yoder B, Gordon M, Barth W. Late preterm birth. Does the changing obstetric paradigm alter the epidemiology of respiratory complications? Obstet Gynecol 2008;111:814-22.

15. Bastek J, Sammel M, Paré E, Srinivas S, Posencheg M, Elovitz M. Adverse neonatal outcomes: examining the risks between preterm, late preterm, and term infants. Am J Obstet Gynecol 2008;199:367.e1-8.

16. Saigal S, Doyle L. Preterm birth 3. An overview of mortality and sequelae of preterm birth from infancy to adulthood. Lancet 2008;37:261-9.

17. Donoso E. Editorial. Rev Chil Obstet Ginecol 2004;69:195-6.

18. Mally $P$, Bailey $S$, Hendricks-Muñoz K. Clinical issues in the management of late preterm infants. Curr Probl Pediatr Adolesc Health Care 2010;40:218-33.

19. Bastek J, Srinivas S, Sammel M, Elovitz M. Do neonatal outcomes differ depending on the cause of preterm birth? A comparison between spontaneous birth and iatrogenic delivery for preeclampsia. Am J Perinatol 2010;27:163-70. 